\title{
1 Experimental Evolution of Cell Shape in Bacteria
}

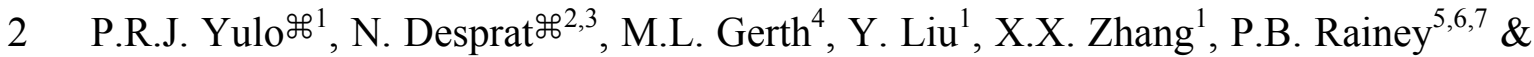

3 H.L. Hendrickson ${ }^{1, \pm}$

4

${ }^{1}$ Institute of Natural and Mathematical Science, Massey University, Auckland.

${ }^{2}$ Laboratoire de Physique Statistique, Ecole Normale Supérieure, PSL Research University; Université Paris Diderot Sorbonne Paris-Cité; Sorbonne Universités UPMC Univeristés Paris 06; CNRS; 24 rue Lhomond, 75005 Paris, France.

${ }^{3}$ Paris Diderot University, 10 rue Alice Domon et Leonie Duquet. 75013 Paris, France.

${ }^{4}$ School of Biological Sciences, Victoria University of Wellington, PO Box 600, 6140, Wellington, New Zealand.

${ }^{5}$ New Zealand Institute for Advanced Study, Massey University, 0745, Auckland, New Zealand

${ }^{6}$ Department of Microbial Population Biology, Max Planck Institute for Evolutionary Biology, Plön, Germany.

${ }^{7}$ Laboratoire de Génétique de l'Evolution, Ecole Superieure de Physique et de Chimie Industrielles de la Ville de Paris (ESPCI Paris Tech), CNRS UMR 8231, PSL Research University, 75231 Paris, France.

HThese authors contributed equally to this work.

¥ Corresponding author, H.Hendrickson@massey.ac.nz

Cell shape is a fundamental property in bacterial kingdom. MreB is a protein that determines rod-like shape, and its deletion is generally lethal. Here, we deleted the mreB homolog from rod-shaped bacterium Pseudomonas fluorescens SBW25 and found that $\triangle m r e B$ cells are viable, spherical cells with a $20 \%$ reduction in competitive fitness and high variability in cell size. We show that cell death, correlated with increased levels of elongation asymmetry between sister cells, accounts for the large fitness reduction. After a thousand generations in rich media, the fitness of evolved $\triangle m r e B$ lines was restored to ancestral levels and cells regained symmetry and ancestral size, while maintaining spherical shape. Using population sequencing, we identified pbp1A, coding for a protein involved in cell wall synthesis, as the primary target for compensatory mutations of the $\triangle m r e B$ genotype. Our findings suggest that reducing elongasome associated PBPs aids in the production of symmetric cells when MreB is absent.

Keywords: Cell shape, Evolution, Experimental Evolution, Coccoid, MreB, Pbp1A, OprD, Single cell analysis, Cell wall synthesis, Asymmetry. 
40 Bacterial cell shape is the result of the coordinated action of a suite of enzymes

41 involved in cell wall construction, DNA segregation and cell division ${ }^{1-6}$. These are

42 highly interdependent processes that can be difficult to genetically disentangle ${ }^{7}$. Cell

43 shape is far from fixed on evolutionary time scales and is a key trait mediating

44 bacterial fitness and adaptation ${ }^{1}$. Rod-like shape is hypothesized to be ancestral in 45 bacteria but a myriad of shapes have successfully developed ${ }^{5,8-10}$. One of the key

46 determinants of rod-like cell shape is MreB, the prokaryotic structural homolog of

$47 \operatorname{actin}^{11,12}$. MreB is the molecular linchpin of rod-like shape and its loss is hypothesized

48 to be either a primary or very early event in the transition between rod-like and 49 spherical cell shape in bacteria ${ }^{5,8-10,13-17}$.

51 MreB acts as a dynamic platform that directs the timing and location of a complex of 52 cell wall elongation enzymes, the 'elongasome' ${ }^{, 18}$ including the bi-functional lateral 53 cell wall synthesis enzyme, Pencillin Binding Protein 1a (PBP1a) ${ }^{19}$. PBP1a and the 54 other members of the elongasome complex move along the inner membrane of rod55 like cells, manufacturing the growing peptidoglycan cell wall ${ }^{20-23,24}$. There is also 56 growing evidence that MreB actively straightens cells during growth by associating 57 with and directing the elongasome to regions of negative curvature in cell walls ${ }^{25,26}$.

58 In addition, MreB disrupting studies, some using A22, demonstrate that the bundled 59 MreB filaments participate in establishing the width and stiffness of the cell while 60 exerting an inward force on the cell wall ${ }^{26-30}$. MreB is also known to have other 61 pleitotropic effects on a range of cellular functions and its loss is frequently lethal in 62 model microbial systems ${ }^{12}$. Some $\Delta m r e B$ mutants can be grown for short periods in 63 heavily supplemented media ${ }^{15}$. In A22 treated cells and in transiently viable $\Delta m r e B$ 64 strains the loss of $m r e B$ function leads to spherical shape and continuous volume increase, lysis and a loss of cell membrane potential ${ }^{5,20,31-33}$.

Previous work has demonstrated the viability of $m r e B$-defective transposon-generated mutants of otherwise rod-shaped Pseudomonas fluorescens SBW2 $5^{34}$. These mutants produce spherical cells in standard Lysogeny Broth (LB) media. The discovery of a nascent spherical phenotype in the absence of MreB in a rod-like bacterium provides 
71 the opportunity to investigate the consequences of MreB loss and the range of compensatory mutations that might restore fitness.

74 Here we demonstrate that deletion of $m r e B(\Delta m r e B)$ in P. fluorescens SBW25 results in viable spherical cells with decreased fitness and highly variable cell size. Evolving this mutant for 1,000 generations in ten independent lineages led to recovery of both WT fitness and cell volume whilst retaining spherical cell shape. Three primary compensatory mutations are studied, two mutations in a PBP (Pencillin Binding Protein) and a separate five-gene deletion. Morphological and single cell time-lapse analysis of strains carrying these mutations demonstrate that these mutations affect

81 lateral cell wall synthesis and septation frequency, reducing sister cell growth asymmetry and proliferation arrest in these cells. Finally, we use comparative genomics of rod-like and spherical cells to infer that PBP loss is a common phenomenon in the evolution of spherical species. Together, our results highlight possible mutational routes by which rod-like cells can adapt their genetic machinery to cope with MreB loss and spherical cell shape.

\section{Methods}

\section{Bacterial strains and culture conditions}

92 Escherichia coli, Neisseria lactamica, and Staphylococcus aureus were grown at $9337^{\circ} \mathrm{C}$, whilst Lactococcus lactis cremoris was grown at $30^{\circ} \mathrm{C}$, and P. fluorescens 94 SBW25 at $28^{\circ} \mathrm{C}$. Antibiotics were used at the following concentrations for E. coli 95 and/or P. fluorescens SBW25: $12 \mu \mathrm{g} \mathrm{ml}^{-1}$ tetracycline; $30 \mu \mathrm{g} \mathrm{ml}^{-1}$ kanamycin; $100 \mu \mathrm{g}$ $96 \mathrm{ml}^{-1}$ ampicillin. Bacteria were propagated in LB.

\section{Strain construction}

99 The $\triangle m r e B$ strain was constructed using SOE-PCR (splicing by overlapping extension 100 using the polymerase chain reaction), followed by a two-step allelic exchange 101 protocol $^{1}$. Genome sequencing confirmed the absence of suppressor mutations. The

102 same procedure was used to reconstruct the mutations from the evolved lines (PBP1a 103 G1450A, PBP1a A1084C, $\triangle$ PFLU4921-4925) into WT-SBW25 and the $\Delta m r e B$ 104 backgrounds. DNA fragments flanking the gene of interest were amplified using two 
105

106

107

108

109

110

111

112

113

114

\section{5}

116

117

118

120

121

122

\section{3}

124

125

126

127

128

129

130

131

132

133

134

135

136

137

138

primer pairs. The internal primers were designed to have overlapping complementary sequences which allowed the resulting fragments to be joined together in a subsequent PCR reaction. The resulting DNA product was TA-cloned into pCR8/GW/TOPO (Invitrogen). This was then subcloned into the pUIC3 vector, which was mobilized via conjugation into SBW25 using pRK2013. Transconjugants were selected on LB plates supplemented with nitrofurantoin, tetracycline and X-gal. Allelic exchange mutants identified as white colonies were obtained from cycloserine enrichment to select against tetracycline resistant cells, and tetracycline sensitive clones were examined for the deletion or mutations using PCR and DNA sequencing.

\section{Evolution Experiment}

Ten replicate populations of the $\triangle m r e B$ strain were grown in $5 \mathrm{~mL}$ aliquots of LB broth at $28^{\circ} \mathrm{C}$ with shaking at $180 \mathrm{rpm}$. Every $24 \mathrm{~h}, 5 \mu \mathrm{L}$ was transferred to fresh media. Every 5 days, samples of each population were collected and stored at $-80^{\circ} \mathrm{C}$ in $15 \%(\mathrm{v} / \mathrm{v})$ glycerol. The number of generations per transfer changed over the course of the experiment but is roughly ten generations per night and $\sim 1,000$ generations (100 transfers) were performed.

\section{Competitive fitness assay}

Competitive fitness was determined relative to SBW25 marked with GFP. This strain was constructed using the mini-Tn7 transposon system, expressing GFP and a gentamicin resistance marker in the chromosome (mini-Tn7(Gm)PrrnB P1 gfp-a) ${ }^{2}$.

Strains were brought to exponential phase in shaken $\mathrm{LB}$ at $28^{\circ} \mathrm{C}$ before beginning the competition. Competing strains were mixed with SBW25-GFP at a 1:1 ratio by adding $150 \mathrm{uL}$ of each strain to $5 \mathrm{~mL} \mathrm{LB}$, then grown under the same conditions for 3 hours. Initial ratios were determined by counting 100,000 cells using flow cytometry (BD FACS Diva II). Suitable dilutions of the initial population were plated on LBA plates to determine viable counts. The mixed culture was diluted 1,000-fold in LB, then incubated at $28^{\circ} \mathrm{C}$ for 24 hours. Final viable counts and ratios were determined as described above. The number of generations over 24 hours of growth were determined using the formula $\ln$ (final population/initial population)/ $\ln (2)$, as previously described ${ }^{3}$. Selection coefficients were calculated using the regression model $s=[\ln (\mathrm{R}(\mathrm{t}) / \mathrm{R}(0))] /[\mathrm{t}]$, where $\mathrm{R}$ is the ratio of the competing strain to SBW25- 
139 GFP, and $t$ is the number of generations. Control experiments were conducted to 140 determine the fitness cost of the GFP marker in SBW25. For each strain, the 141 competition assay was performed with a minimum of 3 replications. WT SBW25 had 142 a relative fitness of 1.0 when compared to the marked strain, indicating that the GFP 143 insert is neutral, and that the SBW25-GFP strain was a suitable reference strain for 144 this assay.

\section{Microscopy}

147 Cells from liquid culture. Cells were routinely grown in LB, and harvested at log 148 phase $\left(\mathrm{OD}_{600}\right.$ 0.4). Viability assays were conducted using the LIVE/DEAD BacLight 149 Bacterial Viability Kit (Thermo Fisher). Viability was measured as the proportion of 150 live cells in the total population (live/(live + dead)). Nucleoid staining was done using 151 the DAPI nucleic acid stain (Thermo Fisher) following the manufacturer's protocols.

153 Time-lapse on agarose pads. Strains were inoculated in LB from glycerol stocks and 154 shaken overnight at $28^{\circ} \mathrm{C}$. The next day, cultures were diluted $10^{2}$ times in fresh LB 155 and seeded on a gel pad (1\% agarose in LB). The preparation was sealed on a glass 156 coverslip with double-sided tape (Gene Frame, Fischer Scientific). A duct was cut 157 through the center of the pad to allow for oxygen diffusion into the gel. Temperature 158 was maintained at $30^{\circ} \mathrm{C}$ using a custom-made temperature controller ${ }^{35}$. ( Bacteria were 159 imaged on a custom built microscope using a 100X/NA 1.4 objective lens (Apo-ph3, 160 Olympus) and an Orca-Flash4.0 CMOS camera (Hamamatsu). Image acquisition and 161 microscope control were actuated with a LabView interface (National Instruments). 162 Typically, we monitored 10 different locations; images were taken every $5 \mathrm{~min}$ in 163 correlation mode ${ }^{36}$. Segmentation and cell lineage were computed using a MatLab 164 code implemented from Schnitzcell ${ }^{37}$. Bacteria were tracked for 3 generations.

166 Scanning Electron Microscopy (SEM). Cells were grown in LB, and harvested at $167 \log$ phase. Cells were fixed in modified Karnovsky's fixative then placed between two 168 membrane filters $(0.4 \mu \mathrm{m}$, Isopore, Merck Millipore LTD) in an aluminum clamp. 169 Following three washes of phosphate buffer, the cells were dehydrated in a graded170 ethanol series, placed in liquid $\mathrm{CO}_{2}$, then dried in a critical-point drying chamber. The 171 samples were mounted onto aluminum stubs and sputter coated with gold (BAL-TEC 
172 SCD 005 sputter coater) and viewed in a FEI Quanta 200 scanning electron

173 microscope at an accelerating voltage of $20 \mathrm{kV}$.

174

175 Image analysis

176 Compactness and estimated volume measurements of cells from liquid culture.

177 The main measure of cell shape, compactness or $\mathbf{C}$, was computed by the CMEIAS

178 software as: $(\sqrt{ } 4$ Area $/ \pi) /$ length. Estimated volume or $\mathbf{V}_{\mathbf{e}}$ was estimated with different

179 formula, according to cell compactness, for spherical cells that have a compactness $\geq$

$1800.7, \mathbf{V}_{\mathbf{e}}$ was computed using the general formula for spheroids: $v=4 / 3 \pi(\mathrm{L} / 2)(\mathrm{W} / 2)^{2}$,

181 where $\mathrm{L}=$ length and $\mathrm{W}=$ =width. $\mathbf{V}_{\mathbf{e}}$ of rod-shaped cells, defined as having a

182 compactness value $\leq 0.7$, were computed using the combined formulas for cylinders

183 and spheres: $\mathbf{V}_{\mathbf{e}}=\left(\pi(\mathrm{W} / 2)^{2}(\mathrm{~L}-\mathrm{W})\right)+\left(4 / 3 \pi(\mathrm{W} / 2)^{3}\right)$.

184

185

186

187

188

189

190

191

192

193

194

195

196

197

198

199

200

201

202

203 Growth asymmetry. For all sister cell pairs, we computed the asymmetry as the

204

Cell size, elongation rate, and division axis of cells on agarose pads. Cell size was computed as the area of the mask retrieved after image segmentation. The elongation axis is given by the major axis of the ellipse that fits the mask of the cell. Division axis is the computed by comparing the elongation axis between mother and sister cell, through the following formula: $|\sin \theta|$, where $\theta$ is the angle between mother and sister cell. We measured the elongation rate of individual bacteria by fitting the temporal dynamics of cell area with a mono-exponential function. The elongation rate is then given by the rate of the exponential. To obtain the intrinsic cell size and disentangle it from the variability associated to asynchrony in the cell cycle, cell size was measured at cell birth, i.e. right after septation. Cell size was then normalized to the size of the WT strain.

Proliferation probability. For the first and second generations, we computed the proliferation probability as the capability of progressing through the cell cycle and dividing. Bacteria that do not grow or stop elongating before dividing are classified as non-proliferating. For all non-proliferating bacteria, we confirmed that no division occurs for the next 5 hours.

contrast in cell elongation given by: $\left|\frac{r_{2}-r_{1}}{r_{1}+r_{2}}\right|$, where $r_{1,2}$ are the elongation rate of the 
205 two sisters measured for the second generation. We then computed the population

206 average on the sub-population that proliferates in order to avoid trivial bias due to cell

207 proliferation arrest of one of the two sister cells.

209 Protein sequence alignment and modeling

210 Protein sequences were obtained from NCBI BLAST (http://blast.ncbi.nlm.nih.gov)

211 and The Pseudomonas Genome Database ${ }^{38}$, and aligned using MEGA7 ${ }^{39}$. The

212 sequence alignment was visualised using ESPript 'Easy Sequencing in PostScript' ${ }^{40}$.

214 Protein visualisation was done on Visual Molecular Dynamics (VMD) ${ }^{41}$ using the

215 crystal structure of Acinetobacter baumannii PBP1a in complex with Aztreonam as 216 the base model, which shares a $73 \%$ sequence identity $(E$ value $=0.0)$ to the PBP1a of 217 P. fluorescens SBW25. Sequences were aligned, and locations of the mutations in the 218 evolved lines were mapped in the corresponding regions. The PDB file was 219 downloaded from the RCSB Protein Data Bank (www.rcsb.org) using PDB ID 3UE0.

\section{Results}

\section{MreB deletion in $\boldsymbol{P}$. fluorescens SBW25 generates viable spherical cells}

$223 \Delta m r e B$ cells to be spherical and display a highly variable cell size and shape 224 compared to the WT strain in phase contrast and SEM (Fig. 1A). The $\triangle m r e B$ strain is 225 viable with approximately $82.5 \%( \pm 7.9 \%)$ live cells compared to WT at $95.2 \%$ $226( \pm 1.2 \%)$ (Fig. 1B). Relative fitness in pairwise competition assays demonstrates that 227 the $\triangle m r e B$ strain has a markedly lower relative fitness of $0.78( \pm 0.02)$ compared to 228 the WT (Fig. 1C) ${ }^{42}$. The $\Delta m r e B$ strain had a slower generation time of $65 \mathrm{~min}$ (WT, $22945 \mathrm{~min}$ ), prolonged lag phase, and lower maximum yield (Supp. Fig. 1).

231 The $m r e B$ gene was ectopically expressed from the $\operatorname{Tn} 7$ site near the $g \operatorname{lm} S$ region of 232 the $\Delta m r e B$ strain completely restored WT morphology, viability, and relative fitness 233 in the $\Delta$ mreB cells with slightly delayed growth (longer lag) (Supp. Fig. 2).

234 Therefore the morphological effects seen in $\triangle m r e B$ are considered to be due solely to 235 loss of MreB. 
237 To quantify variability in size and shape we performed a principle components

238 analysis of the shape metrics (CMEIAS software package) ${ }^{43,44}$ which motivated a 239 focus on a metric called compactness ${ }^{4}$, a measure of the circularity of the cell's 240 outline. A compactness of 1.0 is circular whilst values below 0.7 are more typical of 241 rod-shaped cells. For our purposes, cells with an average compactness of 1.0 to 0.8 , 242 before visible septation initiation are considered to be "spherical". The projected cell 243 outlines were used to estimate volume, $\left(\mathbf{V}_{\mathbf{e}}\right)$ (see Material and Methods) and plotted 244 each cell's $\mathbf{V}_{\mathbf{e}}$ vs compactness for both WT and $\Delta m r e B$ cells (Fig. 1D).

246 WT cells have a small $\mathbf{V}_{\mathbf{e}}$ range and a negative correlation between $\mathbf{V}_{\mathbf{e}}$ and 247 compactness, reflecting the linear elongation and regular cell division of rod-shaped 248 cells. In contrast, the $\triangle m r e B$ strain exhibits large spherical to ovoid cells (, with a 249 wide distribution of $\mathbf{V}_{\mathbf{e}}$ ranging from $1.12 \mathrm{um}^{3}$ to $\sim 90 \mathrm{um}^{3}$, averaging $20.65 \mathrm{um}^{3}$ $250\left( \pm 16.17 \mathrm{um}^{3}\right)$. Spherical $\triangle m r e B$ cells initiate septation at a wide range of volumes 251 from $10 \mathrm{um}^{3}$ to $90 \mathrm{um}^{3}$, indicating that the relationship between cell size and division 252 is lost in $\triangle m r e B$ cells (see lower compactness cells in Fig. 1D).

As cell volume increases, DNA content might also be expected to increase if DNA replication continues irrespective of division frequency. Increased DNA content and 256 spherical cell shape are both predicted to further perturb cell division ${ }^{45,46}$. WT and $257 \triangle m r e B$ cells were stained with a nucleic acid stain (FITC) to label DNA and subjected 258 to flow cytometry. In both strains DNA content scaled with cell size as measured by 259 Forward Scatter Area (FSC-A). The largest $\triangle m r e B$ cells have many times the DNA 260 content of WT cells, scaling roughly with volume (Fig. 1E, Supp. Fig. 3) indicating 261 that DNA replication continues irrespective of cell size. In addition, WT cells 262 observed by time-lapse, orientation of the division plane is consistent across divisions $263(|\sin (\phi)|=0)$. In contrast, in the $\triangle m r e B$ population, septa positioned perpendicularly 264 relative to the last plane at each generation $(\mid(\sin (\phi) \mid=1)$ (Fig. 1F, Supp. Fig. 4B). The 265 change from maintaining septation angles to alternating septation suggests that DNA 266 segregation (prior to septation) is perturbed in cells that have lost rod-like shape. This 267 is consistent with similar results obtained from E. coli treated with the MreB inhibitor, $268 \mathrm{~A} 22^{47}$. 
271 Having established that we have a viable $\triangle m r e B$ in $P$. fluorescens SBW25 an

272 investigation into how this strain adapts to the challenge of MreB loss, was conducted

273 using an experimental evolution approach to select for mutants that restore fitness.

274 After 1,000 generations of evolution (Fig. 2A) the final evolved populations displayed

275 both relative fitness (Fig. 2B) and growth dynamics that were similar to the WT

276 (Supp. Fig. 5). However, the evolved cells remained spherical in shape (Fig. 2D,

277 Supp. Fig. 6). The size, however, as measured by Ve had decreased to roughly that of

278 the ancestral cells (Fig. 2D). The Ve of the evolved lines does not overlap with the

$279 \triangle m r e B$ population (Fig. 1D), evidence that these evolved cells present a new

280 phenotype and are not a subset of the spherical $\triangle m r e B$ ancestor. These newly evolved

281 spherical cells are most similar in cell shape, particularly at septation, to species like

282 Lactococcus lactis cremoris or Neisseria lactamica and other spherical bacterial

283 species that still undergo some elongation prior to division, not like Staphylococcus

284 aureus (Supp. Fig 11) ( $^{48}$. The latter experience rapid division as nearly perfect spheres

285 (Supp. Fig. 11) ${ }^{49}$.

287 In order to understand the dynamics of the fitness recovery the frozen evolved 288 populations were resuscitated at various time points and competed these pairwise 289 against a GFP labeled WT ancestor (Fig. 2C). The fitness increase during evolution 290 occurred rapidly: after only 50 generations of growth, the evolved lines had an 291 average competitive fitness score of $0.92( \pm 0.01)$. This increased to an average fitness 292 of $0.97( \pm 0.02)$ by the end of the experiment.

294 Identifying mutations compensating for costs arising from deletion of mre $B$.

295 The rapid fitness increase observed indicates that a small number of mutations arose 296 early and swept through the populations of poorly competing $\Delta m r e B$ cells. In order to 297 identify these mutations, we conducted population sequencing at 500 and 1,000 298 generations and reference mapped these reads to the P. fluorescens SBW25 genome 299 (GCA_000009225.1) to an average read depth of 100 fold. We identified several 300 mutations affecting open reading frames that were found in over $75 \%$ of the sequence 301 reads in several evolved lines (detailed, Supp. Table 1). A single gene, pbpla had 302 independent mutations in multiple lines. Representative pbpla mutations from lines 1 , 3034 were chosen for further study. Line 7 had a five-gene deletion that included the 304 oprD homolog which was also chosen for further analysis. 
306 The $\operatorname{pbp} 1 A$ gene (PFLU0406) encodes the major Class A penicillin-binding protein

307 responsible for the final steps of peptidoglycan synthesis. PBP1a proteins are key

308 components of peptidoglycan synthesis machinery in the cell wall elongation

309 complexes and are associated with the MreB cytoskeleton in rod-like cells ${ }^{5}$.

311 This PBP1a contains three known domains (Fig. 3A). Structure mapping of the

312 mutations demonstrates that the mutation in Line 1 occurred in a well-conserved 313 region in the transpeptidase (TP) domain, proximal to the active site (Supp. Fig. 7).

314 Similar mutations in Steptococcus pneumoniae ${ }^{50}$ cause a loss of function in this

315 domain. The mutation in Line 4 took place in the oligonucleotide/oligosaccharide

316 binding (OB) domain ${ }^{6}$. These will be referred to from hereon as the pbpla Line 1 and

317 Line 4 mutations respectively. In order to determine the effects of these mutations on

318 cell shape and growth, these separately reconstructed in the WT and $\Delta m r e B$ 319 backgrounds.

321 The $\triangle m r e B$ pbp $1 A$ mutation strains remained spherical to ovoid, near WT volume and 322 DNA content (Fig. 3D, Supp. Fig. 9). These cells also retained the shorter generation 323 times (48 min), growth dynamics (Supp. Fig. 8) and relative fitness of the evolved 324 line populations (Fig. 3C), suggesting that the $p b p 1 A$ mutations are each sufficient to 325 both restore WT fitness and to recapitulate the major phenotypes of evolved lines 1 326 and 4.

327 The function of PBP1A in the ancestral strain and therefore the presence of MreB, is 328 not well studied. The same mutations were therefore reconstructed in the WT 329 background. The WT pbpla mutations also had generation times, growth curves 330 (Supp. Fig. 7) and relative fitness measures similar to WT (Fig. 3C).

332 The major phenotypic difference in the presence of the pbpla mutations was that both 333 the Pbpla TP and $O B$ mutation reconstructions had rod-like cells that are significantly 334 narrower in cell widths ( $0.89 \mathrm{um} \pm 0.07,0.94 \mathrm{um} \pm 0.05$ respectively) compared to 335 WT $(1.00 \mathrm{um} \pm 0.06)(\mathrm{p}=<0.001)$. This resulted in smaller cell volumes (Fig. $3 \mathrm{E}$ and 336 Supp. Fig. 9). This decrease in cell width as a result of an amino acid change near the 337 transpeptidase domain of an elongasome-component is evidence that this mutation 338 decreases the function of PBP1a, likely by interfering with transpeptidase function 
339 (Fig. 3E in Blue). The similar phenotype conferred by the OB domain indicates that 340 these domains act similarly in contributing to cell width (Fig. 3E in Green). The cell 341 size decrease also corresponded with a slight decrease in DNA content (Supp. Fig. 3).

342 The production of thinner cells, is consistent with previous work on the effects of 343 PBP1a function loss in both B. subtilis and in E. coli ${ }^{51-54}$. Based on the positions of 344 the respective mutations, and their resulting phenotypes in WT cells, we interpret 345 these results to indicate that either of these $\operatorname{pbplA}$ mutations can reduce lateral cell 346 wall synthesis, resulting in smaller cells when MreB is present.

348 The other major mutation identified in the evolved lines was a five-gene deletion 349 (PFLU4921-4925) in evolved Line 7 (Fig. 3B, Supp. Table 1). The deletion contains 350 three hypothetical proteins, a cold shock protein (PFLU4922, encoding CspC), and an 351 outer membrane porin, PFLU4925 which encodes OprD. The latter is responsible for 352 the influx of basic amino acids and some antibiotics into the bacterial cell ${ }^{7}$. This 353 deletion was constructed and characterised in the $\Delta m r e B$ and WT backgrounds.

The $\triangle m r e B$ five-gene deletion strain had a generation time and growth dynamics similar to WT, with an additional extended lag time (Supp. Fig. 8). The viability and relative fitness were also highly similar to WT (Fig. 3C). The cells were spherical 358 with an averge Ve of $5.32 \mathrm{um}^{3}( \pm 3.18)$ (Fig. 3D and Supp. Fig. 8). As in the pbp1A 359 Line 1 and Line 4 mutations, DNA content was also decreased compared to the $360 \triangle m r e B$ ancestor (Supp. Fig. 3). In addition, the five-gene deletion produces cell 361 division defects in $25.61 \%( \pm 6.42 \%)$ of these cells, manifesting as septation defects 362 and connected clumps of spherical cells (Fig 3D, Supp. Fig. 9).

364 In the WT strain, the five-gene deletion produced rod shaped cells with growth 365 characteristics similar to the WT strain (Supp. Fig 8). These cells were however 366 significantly thinner than WT (width $=0.74 \mathrm{um} \pm 0.06, \mathrm{p}=<0.001$ ) and had a smaller 367 average Ve of $2.47 \mathrm{um}^{3}( \pm 1.18)$ (Fig. 3E and Supp. Fig. 8). As in the $\Delta m r e B$ 368 background, a sub-population exhibits a filamenting phenotype occurring in $20 \%$ ( \pm $3694 \%$ ) of the population. The five-gene deletion strains were the only ones that showed 370 evidence of dispersed DNA between incomplete septa in DAPI staining (Supp. Fig 371 S10). Intriguingly, clinically isolated Pseudomonas with oprD deletions have 372 significant changes in the regulation of the MinCD system ${ }^{55}$. In closely related model 
systems MinCD, acts to negatively affect septal placement by poles and accumulating as a result of cell shape asymmetry ${ }^{17,56-58}$. The connection between OprD and MinCD in Pseudomonas merits further investigation but $\operatorname{oprD}$ loss may mitigate large cell size and increase fitness in $\triangle m r e B$ by retuning septation frequencies. This would also imply that the viable $\triangle m r e B$ cells lack proper the geometry required to support MinCD oscillations ${ }^{59}$, resulting in erratic septation and driving large cell size.

\section{Sister Cell Asymmetry at the Single Cell Level}

In order to determine the basis of the fitness cost of the mreB deletion, we, we conducted single cell experiments in the reconstructed mutants and representative evolved clones from lines $1,4 \& 7$. Time-lapse microscopy was used to track individual cells through subsequent generations to measure size, elongation rate, division axis and shape for each cell as well as their capacity to produce two daughters ${ }^{36,60}$.

All reconstructed strains except the strain that ectopically expresses mreB (closed grey square), have a reduced rate of cell wall synthesis relative to the WT (Fig. 4A) but all are higher than the ancestral $\triangle m r e B$ strain. Cell elongation rates are higher in the presence of MreB in the pbplA Line 4 mutant, but not the Line 1 mutant, suggesting that the transpeptidase domain mutation may affect the degree to which MreB stimulates synthesis ${ }^{61}$.

In addition, single cell experiments measured that a fraction of cells underwent persistent proliferation arrest on solid media, even after five hours of observation (Fig. 4B). Tracking pairs of dividing cells coming from the same mother revealed that they experience unequal rates of cell wall synthesis, or 'growth asymmetry' (Fig. 4B). A strong correlation is observed between proliferation arrest and growth asymmetry in our reconstructed mutation strains and representative clones. In strains that had higher growth asymmetry, more proliferation arrest was observed (Fig 4D). This increased growth asymmetry might either initiate proliferation arrest or both features may be symptoms of another attribute of these cells such as cell size or defects in DNA segregation driven by cell shape and septum aberrations ${ }^{46,62}$. 
406 Accordingly, cells that have lost MreB are able to find a new equilibrium by 407 decreasing elongation synthesis ( $p b p 1 A$ mutations) or possibly modulating septation 408 associated synthesis (the oprD inclusive deletion). Either serves to increase the 409 relative proportion of synthesis at the septum, and decrease elongasome associated 410 synthesis. The advantage gained through these adjustments in response to MreB loss 411 hint at a previously unrecognized role of MreB in ensuring the equal partitioning of 412 the elongasome components before and after cell division.

\section{Recapitulating spherical shape evolution}

415 These experiments demonstrate that either a decrease in activity in a PBP in the 416 elongasome or a five-gene deletion that includes $\operatorname{or} D$ allow a rebound in fitness 417 when $m r e B$ is lost. It was previously reported that coccoid bacterial species have 418 lower estimated numbers of PBPs based on estimates from a biochemical function 419 assay $^{63}$. We were therefore interested in whether comparative genomics of completely sequenced bacteria bore this pattern out as well. We therefore selected 26 bacterial

421 species pairs in which one member has maintained rod-like shape and the other has

422 become spherical and compared the abundance of the homologues of the genes

423 implicated in our evolution experiment; MreB, PBPs and OprD homologs. OprD

424 homologs were too rare across species to analyze. However, we observed a significant

425 relationship between coccoid lineages that had lost MreB and a decrease in the 426 number of PBP homologs (avg. PBPs in rods $=9.22 \pm 5.15$; spheres $=3.89 \pm 2.65$;

427 difference: $p=<0.001)$. From this we infer that species that have naturally evolved 428 from rod-like to spherical shape tend to have lost both $m r e B$ and approximately half 429 of their PBP genes.

431 Reshaping a rod-like pseudomonad to be a spherical cell

432 P. fluorescens SBW25 is a rod-like bacterium that can be reshaped into a rapidly 433 growing spherical cell in as little as two mutational steps, the deletion of mreB and 434 either a single amino acid changing mutation in $p b p 1 A$ or an $\operatorname{oprD}$ inclusive deletion. 435 The reason that this strain is tolerant of MreB loss is not currently known but a 436 separate paralog does not exist in this strain.

438 The loss of MreB from the ancestral SBW25 causes extremely large cells with 439 multiple chromosomes (Fig. 1D,E) with highly irregular septation. In addition, sister 
440 cells elongate perpendicularly to mother cells, across cell divisions (Fig 1E-F),

441 consistent with MreB disruption experiments using $\mathrm{i} i$ and $P$. aeriginosa ${ }^{31,64}$. Both cell

442 wall synthesis and DNA replication are continuous in these cells (Fig. 1E and Fig.

443 4A) meaning that large cell size is the result of a reduction in septation frequency,

444 maybe due to the loss of the ordered relationship between septation and DNA

445 segregation in spherical cells ${ }^{46}$.

447 The $\triangle m r e B$ population had high levels of cell wall synthesis asymmetry and while 448 either of the $\operatorname{pbp} 1 A$ mutations increased this symmetry, the five-gene deletion did not 449 (Fig. 4D). This increase in symmetry suggests that the distribution of active 450 elongasomes may be disorganised in cells lacking MreB and that this disorganization 451 is reduced when $\operatorname{pbplA}$ is mutated $^{52}$ (Fig $4 \mathrm{C}$ ). This raises the possibility that symmetry 452 in cell synthesis is maintained in these cells by continued septal cell wall synthesis. 453 While this is consistent with models of other spherically shaped cells in which much 454 of the cell wall synthesis further investigation of cell wall synthesis is required to 455 support or refute this hypothesis ${ }^{65-67}$.

\section{Implications for the evolution of spherical cell shape}

458 The wide array of cell shapes and sizes observed in the eubacteria have arisen from an 459 ancestral rod-like cell shape ${ }^{2,68-70}$ Coccoid or spherical cells are the product of a 460 degradation of this shape ${ }^{14,71}$. The transition to spherical cell shape has taken place 461 independently many times ${ }^{8,72-74}$ and is associated with $m r e B$ loss, possibly as an early 462 event $^{48,49}$.

464 This study uncovers separate compensatory mutations that allow rapid fitness 465 recovery after MreB loss. If MreB loss is a common early event in coccus evolution 466 then there are likely to be both genetic and environmental contexts that favor this 467 state $2,9,14,71$. One possibility is that the transient increase in cell size observed in $468 \Delta m r e B$ cells is advantageous in some settings. This hypothesis compels further 469 investigation $^{16}$.

\section{Conclusions}

473 Cell shape is a fundamental property of cells that defines motility, DNA segregation, 
474 replication, nutrient acquisition, waste elimination and predator evasion ${ }^{16}$. $P$.

475 fluorescens SBW25 is a rod-shaped bacterium that is amenable to MreB deletion.

476 The loss of MreB is a non-lethal but deleterious event that leads to irregular, large-

477 sized spherical cells. Further, separate mutations can restore fitness and volume whilst

478 retaining spherical cell shape and these are likely decrease-of-function mutations in

479 the gene encoding elongasome member PBP1A, or a five-gene deletion that includes

480 oprD. These mutations are able to restore symmetry in cell growth between sister

481 cells and decrease cell death. We therefore propose a model of molecular change

482 when MreB is lost, essentially re-storing symmetric cell wall synthesis by relying

483 more heavily on synthesis at the septum. Last, our study implicates a decrease of PBP

484 function, as a general strategy in cells recovering from the loss of MreB and refining

485 spherical cell shape in bacteria.

486

487 Acknowledgements

488

489 We thank Olin Silander for helpful discussions and for his assistance to PRY with

490 principle components analysis of cell shape, Sebastian Schmeier and Saumya

491 Agrawal for bioinformatic analysis and Tim Cooper for helpful comments on the

492 manuscript. Electron Microscopy was provided by Massey University and was

493 performed by Niki Murray, Manawatu Microscopy and Imaging Centre, Massey

494 University, Palmerston North, NZ.

495

496 Figures 

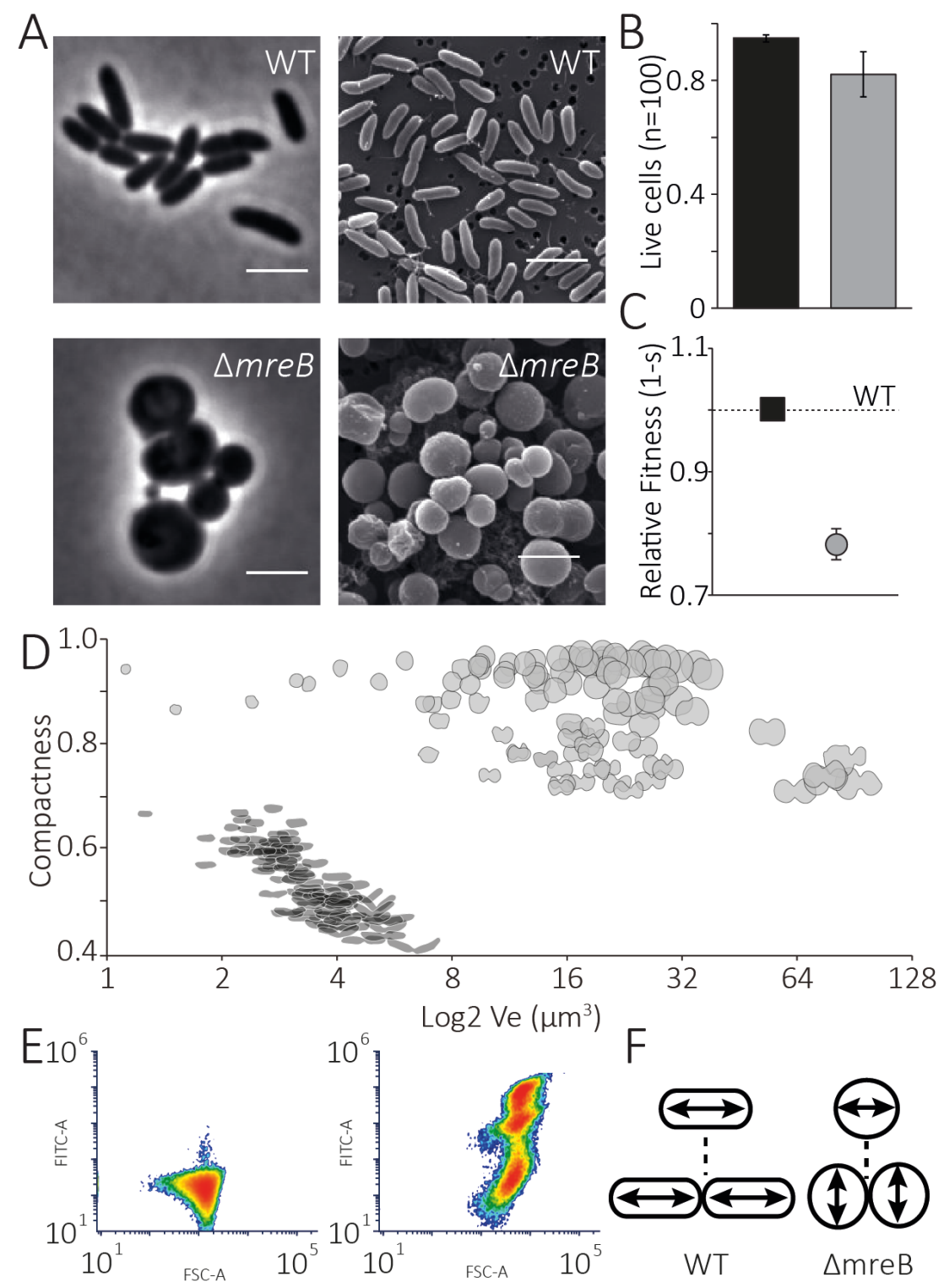

520 Figure 1. Characterisation of WT and $\triangle m r e B$ strains. Figure shows (A)

521 photomicrographs of WT and $\triangle m r e B$. Scale bars, $3 \mu \mathrm{m}$. B) Proportion of live cells in

522 WT (black bar) and $\triangle m r e B$ (grey bar). Error bars represent standard error $(\mathrm{n}=3)$. C)

523 Fitness of WT GFP and the ancestral $\triangle m r e B$ mutant relative to WT when both are in

524 exponential phase during pairwise competition assays. Error bars as in 1B. D) The

525 relationship between cell shape and estimated volume $\left(\mathrm{V}_{\mathrm{e}}\right)$ is represented using

526 compactness, a measure of roundness. One hundred representative cells from each

$527 \mathrm{WT}$ and $\triangle m r e B$ are shown as cell outlines. E) DNA content (FITC-A) is highly

528 correlated with increased cell sizes (FSC-A) in $\triangle m r e B$ but both are limited in WT

529 cells $(n=50,000$ events). F) Diagram of WT cells maintaining a single consistent

530 division plane whilst $\triangle m r e B$ cells alternate division planes by $90^{\circ}$ from one division 531 to the next. 

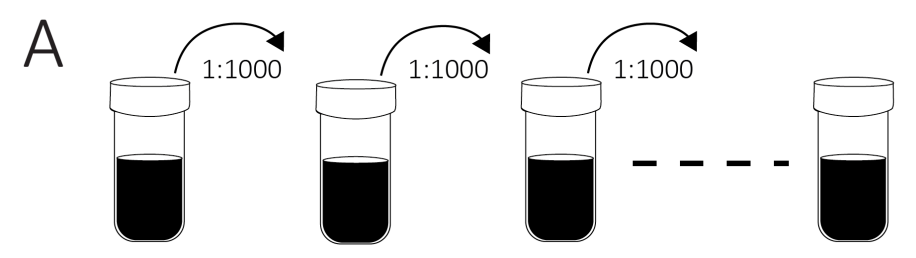

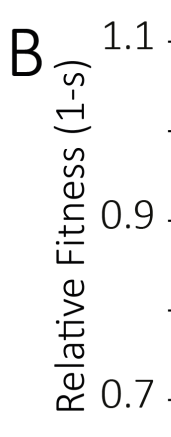

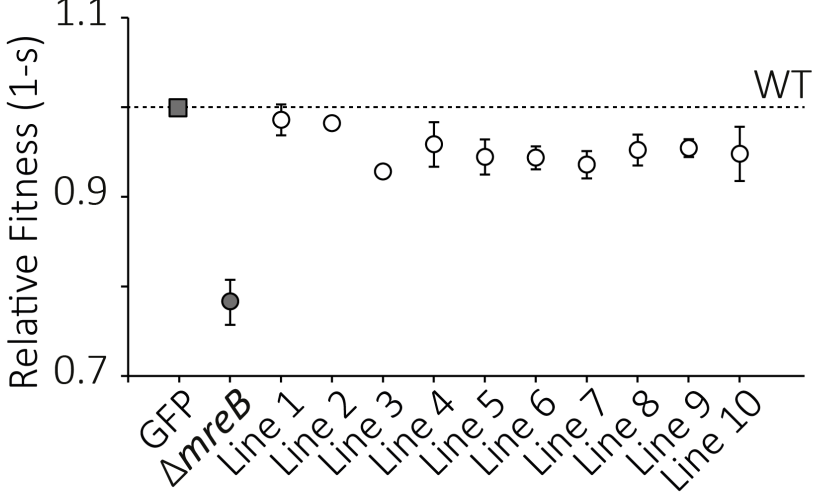

558 Figure 2. Characterisation of Evolved Lines at 1,000 generations. A) Diagram of
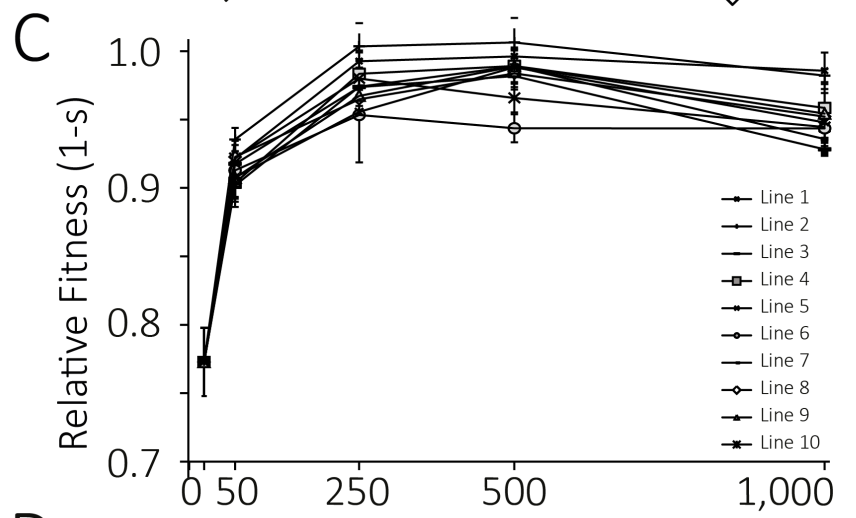

D

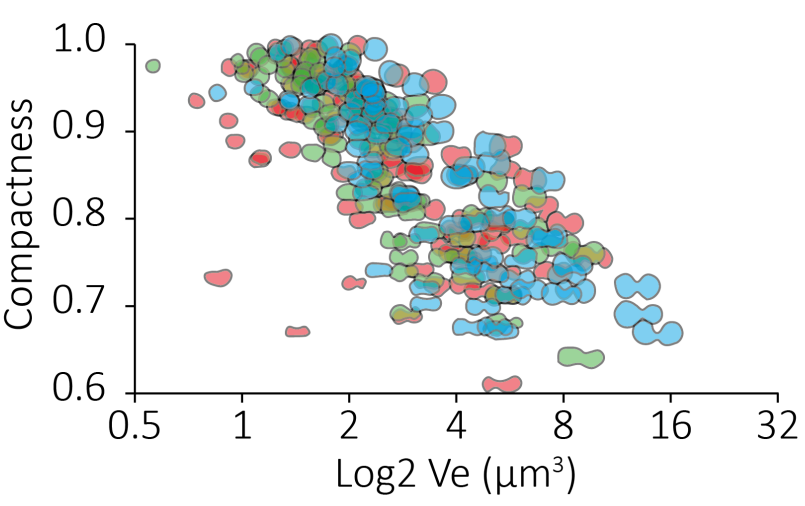

561 line) in pairwise competition experiments. Error bars represent standard error $(\mathrm{n}=3)$.

562 C) Relative fitness of the evolved lines during 1,000 generations of growth. Error bars

563 as in B. D) Cell outlines of three representative evolved lines Line 1 in blue, Line 4 in

564 green and line 7 in red. One-hundred randomly chosen cell outlines from each line are 565 depicted. 


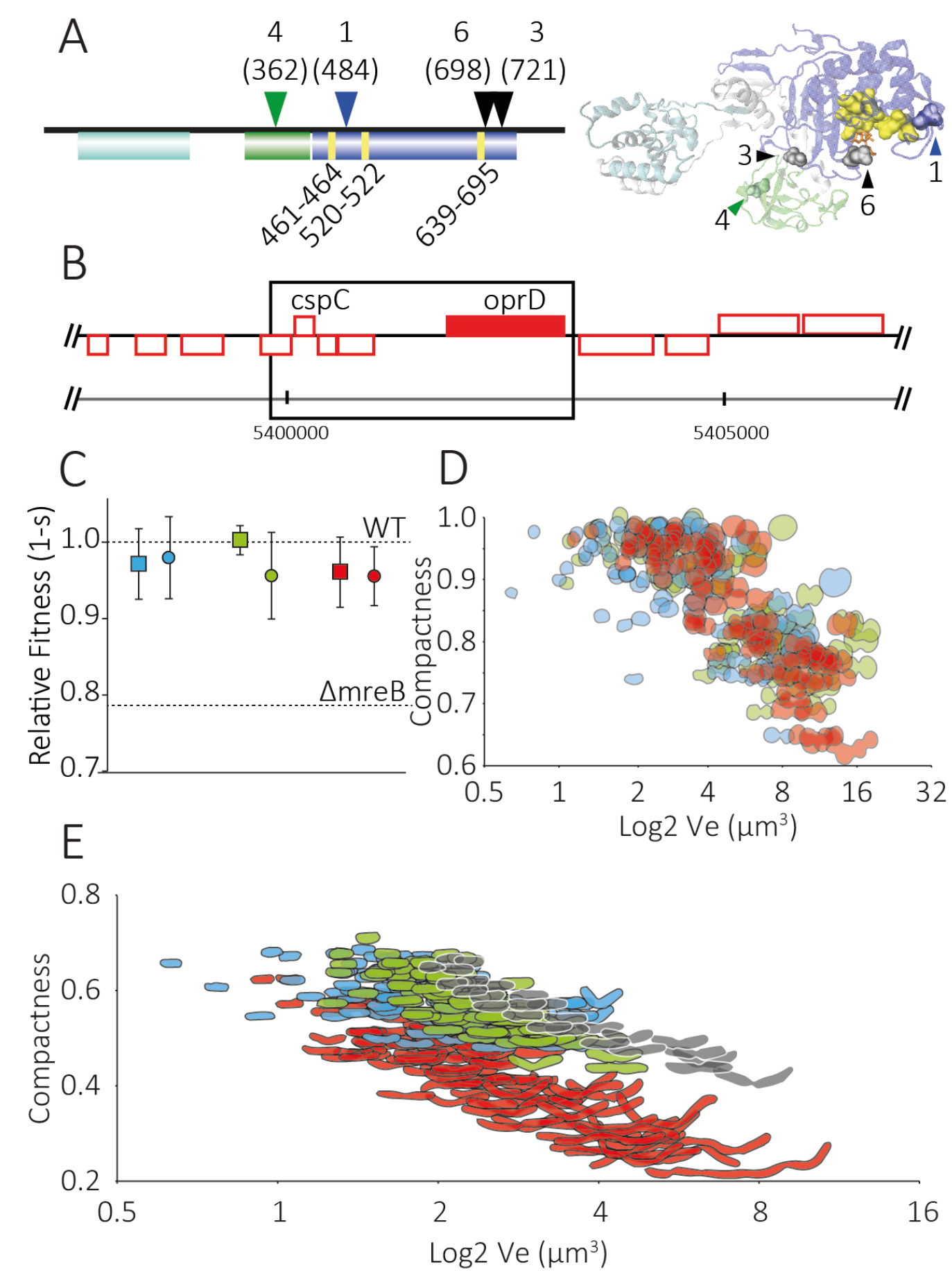

567 Figure 3. Characterisation of the reconstructed mutations in WT and $\Delta m r e B$

568 background. A) Domain map and model of PBP1a (PFLU0406) showing the 2 major

569 active sites; the glycosyltransferase (GT) domain (cyan) and the transpeptidase (TP)

570 domain (blue). The oligonucleotide/oligosaccharide binding (OB) domain is shown in

571 green. The active site of the TP domain is also shown (yellow). The mutations

572 identified are indicated above the map. B) Genome map of oprD inclusive deletion,

573 (PFLU4921-PFLU4925) and surrounding region. Genes with function calls are noted.

574 C) Relative fitness of the three reconstructed mutants in the $\Delta m r e B$ (circles) and WT 
bioRxiv preprint doi: https://doi.org/10.1101/263681; this version posted February 11,2018 . The copyright holder for this preprint (which

was not certified by peer review) is the author/funder, who has granted bioRxiv a license to display the preprint in perpetuity. It is made available under aCC-BY-NC-ND 4.0 International license.

575 (squares) backgrounds. Line 1 reconstruction, PBP1a D484N is shown in blue, Line 4 576 PBP1a T362P is shown in green and the OprD containing deletion reconstruction is

577 shown in red. D) Compactness versus estimated volume (Ve) for reconstruction 578 strains in the $\triangle m r e B$ background, colors as in $1 \mathrm{C}(\mathrm{N}=100)$. E) Compactness versus 579 estimated volume (Ve) of the mutants in the WT background colours and $\mathrm{N}$ as in $\mathrm{C}$ 580 and D. A subset of WT cells are shown in light grey for comparison.

581

582 


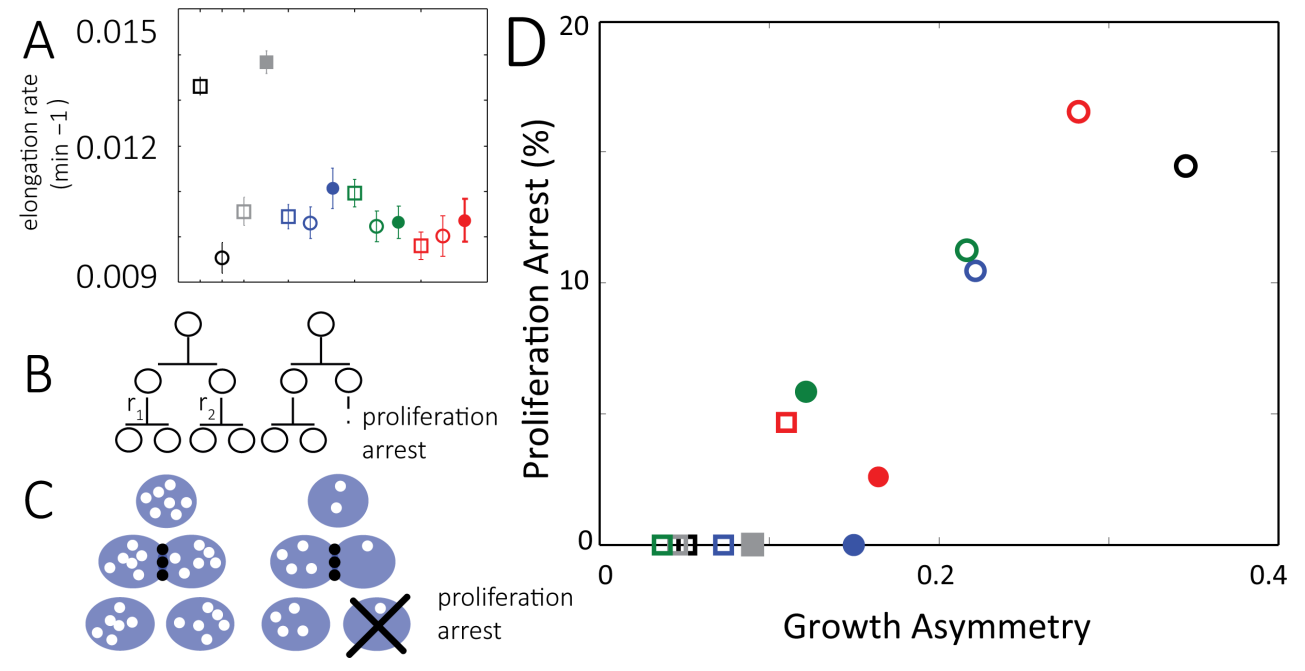

585 Figure 4. Single cell time-lapse measurements of reconstructed mutations. A) Average 586 population elongation rate for WT, reconstructions and evolved cells. Open square data points represent WT or WT and respective mutations (open grey square $=\triangle m r e B$

588 with ectopic $m r e B$ ), open circles are $\triangle m r e B$ or $\triangle m r e B$ and respective mutations and 589 closed symbols are evolved cell lines. Colors as in Fig 3; blue is Line 1 or PBP1a 590 D484N, green is Line 4 or PBP1a T362P, red is Line 7 or OprD inclusive deletion. B) 591 Strategy for analyzing single cell measurements during growth on agarose pads 592 including $r_{1}$ and $r_{2}$. C) Model for relationship between growth asymmetry and 593 proliferation arrest driven by disordered acquisition of cell wall synthesis machinery 594 (white dots). The septum associated PBPs (black circles) at the division plane provide 595 symmetric cell wall synthesis to respective daughter cells. D) Relationship between 596 paired-sister cell proliferation arrest and growth asymmetry in all reconstructions and 597 representative evolved cells $(\mathrm{N}=100$ for each). 
bioRxiv preprint doi: https://doi.org/10.1101/263681; this version posted February 11,2018 . The copyright holder for this preprint (which was not certified by peer review) is the author/funder, who has granted bioRxiv a license to display the preprint in perpetuity. It is made available under aCC-BY-NC-ND 4.0 International license.
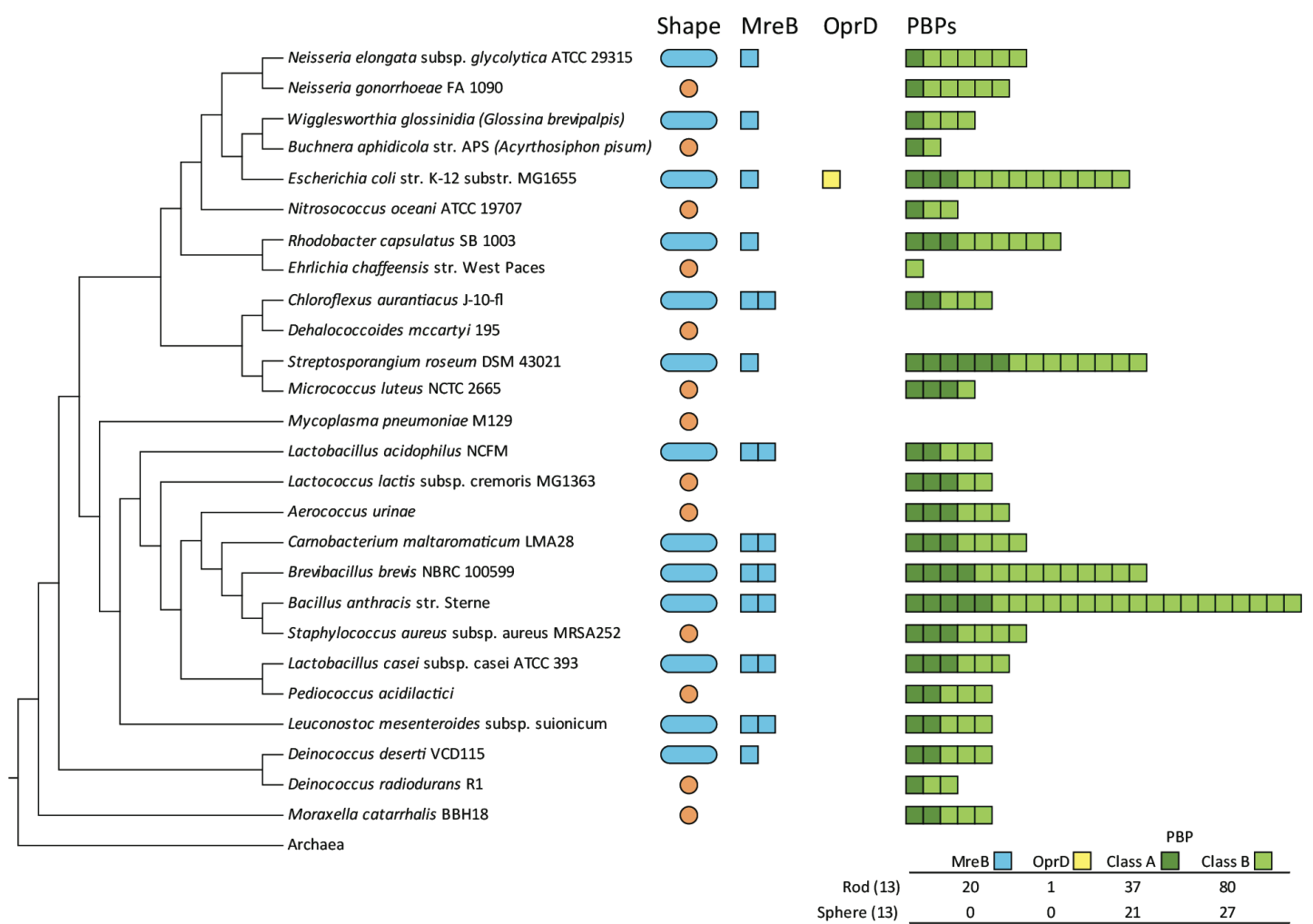

601 Figure 5. Penicillin-Binding Proteins (PBPs) is observed in extant spherically-shaped

602 cells. A selection of 26 paired rod-shaped and spherical cells were analyzed for their

603 PBP and OprD homologs. The genomes of naturally evolved spherical cells have

604 fewer PBPs than rod-shaped species. OprD homologs were rare. 


\section{References:}

1. Young, K. D. Bacterial morphology: why have different shapes? Curr Opin Microbiol 10, 596-600 (2007).

2. $\quad$ Young, K. D. Bacterial shape. Mol Microbiol 49, 571-580 (2003).

3. Young, K. D. Bacterial shape: two-dimensional questions and possibilities. Annu. Rev. Microbiol. 64, 223-240 (2010).

4. Huang, K. C., Mukhopadhyay, R., Wen, B., Gitai, Z. \& Wingreen, N. S. Cell shape and cell-wall organization in Gram-negative bacteria. Proc Natl Acad Sci USA 105, 19282-19287 (2008).

5. Daniel, R. A. \& Errington, J. Control of cell morphogenesis in bacteria: two distinct ways to make a rod-shaped cell. Cell 113, 767-776 (2003).

6. Osborn, M. J. \& Rothfield, L. Cell shape determination in Escherichia coli. Curr Opin Microbiol 10, 606-610 (2007).

7. van Teeffelen, S. et al. The bacterial actin MreB rotates, and rotation depends on cell-wall assembly. Proc Natl Acad Sci USA 108, 15822-15827 (2011).

8. Siefert, J. L. \& Fox, G. E. Phylogenetic mapping of bacterial morphology. Microbiology (Reading, Engl.) 144 ( Pt 10), 2803-2808 (1998).

9. Veyrier, F. J. et al. Common Cell Shape Evolution of Two Nasopharyngeal Pathogens. PLoS Genet. 11, e1005338 (2015).

10. Margolin, W. Sculpting the bacterial cell. Curr Biol 19, R812-22 (2009).

11. Strahl, H., Bürmann, F. \& Hamoen, L. W. The actin homologue MreB organizes the bacterial cell membrane. Nat Commun 5, 3442 (2014).

12. Shaevitz, J. W. \& Gitai, Z. The Structure and Function of Bacterial Actin Homologs. Cold Spring Harbor Perspectives in Biology 2, a000364-a000364 (2010).

13. Tamames, J., González-Moreno, M. \& Mingorance, J. Bringing gene order into bacterial shape. TRENDS in ... (2001).

14. Baidouri, El, F., Venditti, C. \& Humphries, S. Independent evolution of shape and motility allows evolutionary flexibility in Firmicutes bacteria. Nat. ecol. evol. 1, 0009-6 (2016).

15. Formstone, A. \& Errington, J. A magnesium-dependent mreB null mutant: implications for the role of mreB in Bacillus subtilis. 55, 1646-1657 (2005).

16. Young, K. D. The selective value of bacterial shape. Microbiol Mol Biol Rev 70, 660-703 (2006).

17. Pichoff, S. \& Lutkenhaus, J. Overview of cell shape: cytoskeletons shape bacterial cells. Curr Opin Microbiol 10, 601-605 (2007).

18. Szwedziak, P. \& Löwe, J. Do the divisome and elongasome share a common evolutionary past? Curr Opin Microbiol 16, 745-751 (2013).

19. Zhao, H., Patel, V., Helmann, J. D. \& Dörr, T. Don't let sleeping dogmas lie: New views of peptidoglycan synthesis and its regulation. Mol Microbiol (2017). doi:10.1111/mmi.13853

20. Figge, R. M., Divakaruni, A. V. \& Gober, J. W. MreB, the cell shapedetermining bacterial actin homologue, co-ordinates cell wall morphogenesis in Caulobacter crescentus. Mol Microbiol (2004).

21. Ouzounov, N. et al. MreB Orientation Correlates with Cell Diameter in Escherichia coli. Biophysj 111, 1035-1043 (2016).

22. Garner, E. C. et al. Coupled, circumferential motions of the cell wall synthesis machinery and MreB filaments in B. subtilis. Science 333, 222-225 (2011).

23. Cava, F., Kuru, E., Brun, Y. V. \& de Pedro, M. A. Modes of cell wall growth 
differentiation in rod-shaped bacteria. Curr Opin Microbiol 16, 731-737 (2013).

24. Domínguez-Escobar, J. et al. Processive movement of MreB-associated cell wall biosynthetic complexes in bacteria. Science 333, 225-228 (2011).

25. Tropini, C. et al. Principles of Bacterial Cell-Size Determination Revealed by Cell-Wall Synthesis Perturbations. CellReports 9, 1520-1527 (2014).

26. Ursell, T. S. et al. Rod-like bacterial shape is maintained by feedback between cell curvature and cytoskeletal localization. Proc Natl Acad Sci USA 111, E1025-34 (2014).

27. Sun, S. X. \& Jiang, H. Physics of bacterial morphogenesis. Microbiol Mol Biol Rev 75, 543-565 (2011).

28. Tuson, H. H. et al. Measuring the stiffness of bacterial cells from growth rates in hydrogels of tunable elasticity. Mol Microbiol 84, 874-891 (2012).

29. Garner, E. C., Bernard, R., Wang, W., Zhuang, X. \& Rudner, D. Z. Coupled, circumferential motions of the cell wall synthesis machinery and MreB filaments in B. subtilis. Science (2011).

30. Hussain, S. et al. MreB Filaments Create Rod Shape By Aligning Along Principal Membrane Curvature. bioRxiv 1-94 (2017). doi:10.1101/197475

31. Robertson, G. T. et al. A Novel Indole Compound That Inhibits Pseudomonas aeruginosa Growth by Targeting MreB Is a Substrate for MexAB-OprM. Journal of Bacteriology 189, 6870-6881 (2007).

32. Kruse, T., Bork-Jensen, J. \& Gerdes, K. The morphogenetic MreBCD proteins of Escherichia coli form an essential membrane-bound complex. $\mathrm{Mol}$ Microbiol 55, 78-89 (2005).

33. Bendezú, F. O. \& de Boer, P. A. J. Conditional lethality, division defects, membrane involution, and endocytosis in mre and mrd shape mutants of Escherichia coli. Journal of Bacteriology 190, 1792-1811 (2008).

34. McDonald, M. J., Gehrig, S. M., Meintjes, P. L., Zhang, X.-X. \& Rainey, P. B. Adaptive divergence in experimental populations of Pseudomonas fluorescens. IV. Genetic constraints guide evolutionary trajectories in a parallel adaptive radiation. Genetics 183, 1041-1053 (2009).

35. Julou, T., Desprat, N., Bensimon, D. \& Croquette, V. Monitoring microbial population dynamics at low densities. Rev Sci Instrum 83, 074301 (2012).

36. Julou, T. et al. Cell-cell contacts confine public goods diffusion inside Pseudomonas aeruginosa clonal microcolonies. Proc Natl Acad Sci USA 110, 12577-12582 (2013).

37. Locke, J. C. W. \& Elowitz, M. B. Using movies to analyse gene circuit dynamics in single cells. Nature Publishing Group 7, 383-392 (2009).

38. Winsor, G. L. et al. Enhanced annotations and features for comparing thousands of Pseudomonas genomes in the Pseudomonas genome database. Nucleic Acids Res 44, D646-53 (2016).

39. Kumar, S., Stecher, G. \& Tamura, K. MEGA7: Molecular Evolutionary Genetics Analysis Version 7.0 for Bigger Datasets. Mol Biol Evol 33, 1870 1874 (2016).

40. Robert, X. \& Gouet, P. Deciphering key features in protein structures with the new ENDscript server. Nucleic Acids Res 42, W320-4 (2014).

41. Humphrey, W., Dalke, A. \& Schulten, K. VMD: visual molecular dynamics. $J$ Mol Graph 14, 33-8-27-8 (1996).

42. Lind, P. A., Farr, A. D. \& Rainey, P. B. Experimental evolution reveals hidden diversity in evolutionary pathways. Elife (2015). doi:10.7554/eLife.07074.001 
43. Liu, J., Dazzo, F. B., Glagoleva, O., Yu, B. \& Jain, A. K. CMEIAS: A Computer-Aided System for the Image Analysis of Bacterial Morphotypes in Microbial Communities. 41, 173-194 (2001).

44. Folland, I., Trione, D. \& Dazzo, F. Accuracy of Biovolume Formulas for CMEIAS Computer-Assisted Microscopy and Body Size Analysis of Morphologically Diverse Microbial Populations and Communities. Microb. Ecol. 1-15 (2014). doi:10.1007/s00248-014-0410-9

45. Jun, S. \& Mulder, B. Entropy-driven spatial organization of highly confined polymers: lessons for the bacterial chromosome. Proc Natl Acad Sci USA 103, 12388-12393 (2006).

46. Jun, S. \& Wright, A. Entropy as the driver of chromosome segregation. Nat Rev Micro (2010). doi:10.1038/nrmicro2391

47. Begg, K. J. \& Donachie, W. D. Division planes alternate in spherical cells of Escherichia coli. Journal of Bacteriology 180, 2564-2567 (1998).

48. Zapun, A., Vernet, T. \& Pinho, M. G. The different shapes of cocci. FEMS Microbiology Reviews 32, 345-360 (2008).

49. Pinho, M. G., Kjos, M. \& Veening, J.-W. How to get (a)round: mechanisms controlling growth and division of coccoid bacteria. Nature Publishing Group 11, 601-614 (2013).

50. Job, V., Carapito, R., Vernet, T., Dessen, A. \& Zapun, A. Common alterations in PBP1a from resistant Streptococcus pneumoniae decrease its reactivity toward beta-lactams: structural insights. J Biol Chem 283, 4886-4894 (2008).

51. Murray, T., Popham, D. L. \& Setlow, P. Bacillus subtilis cells lacking penicillin-binding protein 1 require increased levels of divalent cations for growth. Journal of Bacteriology 180, 4555-4563 (1998).

52. Kawai, Y., Daniel, R. A. \& Errington, J. Regulation of cell wall morphogenesis in Bacillus subtilis by recruitment of PBP1 to the MreB helix. Mol Microbiol 71, 1131-1144 (2009).

53. Banzhaf, M. et al. Cooperativity of peptidoglycan synthases active in bacterial cell elongation. Mol Microbiol 85, 179-194 (2012).

54. Claessen, D. et al. Control of the cell elongation-division cycle by shuttling of PBP1 protein in Bacillus subtilis. Mol Microbiol 68, 1029-1046 (2008).

55. Skurnik, D., Roux, D. \& Cattoir, V. Enhanced in vivo fitness of carbapenemresistant oprD mutants of Pseudomonas aeruginosa revealed through highthroughput sequencing. in (2013). doi:10.1073/pnas.1221552110//DCSupplemental

56. Shih, Y. L., Kawagishi, I. \& Rothfield, L. The MreB and Min cytoskeletal-like systems play independent roles in prokaryotic polar differentiation. 58, $917-$ 928 (2005).

57. Chien, A.-C., Hill, N. S. \& Levin, P. A. Cell Size Control in Bacteria. 22, R340-R349 (2012).

58. Corbin, B. D., Yu, X.-C. \& Margolin, W. Exploring intracellular space: function of the Min system in round-shaped Escherichia coli. EMBO J 21, 1998-2008 (2002).

59. Halatek, J. \& Frey, E. Highly canalized MinD transfer and MinE sequestration explain the origin of robust MinCDE-protein dynamics. CellReports 1, 741752 (2012).

60. Desprat, N., Richert, A., Simeon, J. \& Asnacios, A. Creep function of a single living cell. Biophysj 88, 2224-2233 (2005).

61. Typas, A., Banzhaf, M., Gross, C. A. \& Vollmer, W. From the regulation of 
peptidoglycan synthesis to bacterial growth and morphology. Nature Publishing Group 10, 123-136 (2011).

62. Bigot, S., Sivanathan, V., Possoz, C., Barre, F.-X. \& Cornet, F. FtsK, a literate chromosome segregation machine. 64, 1434-1441 (2007).

63. Lleo, M. M., Canepari, P. \& Satta, G. Bacterial cell shape regulation: testing of additional predictions unique to the two-competing-sites model for peptidoglycan assembly and isolation of conditional rod-shaped mutants from some wild-type cocci. Journal of Bacteriology 172, 3758-3771 (1990).

64. Bendezú, F. O. \& de Boer, P. A. J. Conditional lethality, division defects, membrane involution, and endocytosis in mre and mrd shape mutants of Escherichia coli. Journal of Bacteriology 190, 1792-1811 (2008).

65. Pérez-Núñez, D. et al. A new morphogenesis pathway in bacteria: unbalanced activity of cell wall synthesis machineries leads to coccus-to-rod transition and filamentation in ovococci. Mol Microbiol 79, 759-771 (2011).

66. Lleo, M. M., Canepari, P. \& Satta, G. Bacterial cell shape regulation: testing of additional predictions unique to the two-competing-sites model for peptidoglycan assembly and isolation of conditional rod-shaped mutants from some wild-type cocci. Journal of Bacteriology 172, 3758-3771 (1990).

67. Monteiro, J. A. O. M. et al. Cell shape dynamics during the staphylococcal cell cycle. Nat Commun 6, 1-12 (2015).

68. Young, K. D. Bacterial Shape: Two-Dimensional Questions and Possibilities. 64, 223-240 (2010).

69. Koch, A. L. Were Gram-positive rods the first bacteria? Trends Microbiol 11, 166-170 (2003).

70. Errington, J. L-form bacteria, cell walls and the origins of life. Open Biology 3, 120143-120143 (2013).

71. Siefert, J. L. \& Fox, G. E. Phylogenetic mapping of bacterial morphology. Microbiology (Reading, Engl.) 144 ( Pt 10), 2803-2808 (1998).

72. Stackebrandt, E. \& Woese, C. R. A phylogenetic dissection of the family micrococcaceae. Curr. Microbiol. 2, 317-322 (1979).

73. Tamames, J., González-Moreno, M., Mingorance, J., Valencia, A. \& Vicente, M. Bringing gene order into bacterial shape. Trends Genet 17, 124-126 (2001).

74. Baidouri, El, F., Venditti, C. \& Humphries, S. Independent evolution of shape and motility allows evolutionary flexibility in Firmicutes bacteria. Nat. ecol. evol. 1, 0009-6 (2016). 